\title{
A CONSISTENT EXPLANATION OF THE ROPER PHENOMENOLOGY
}

\author{
F. Cano and P. González \\ Departamento de Física Teórica and IFIC, \\ Centro Mixto Universidad de Valencia-CSIC \\ 46100 Burjassot (Valencia), Spain
}

\begin{abstract}
We study the electromagnetic transitions of the Roper $N(1440)$ resonance. Our results, when combined with the previously obtained for the mass and the pionic strong decay widths of the Roper, show that within a non-relativistic constituent quark model scheme, a comprehensible understanding of the Roper phenomenology can be achieved. They also seem to support the view of the Roper as a radial excitation of the nucleon, though more experimental data are needed to reach a definitive conclusion.
\end{abstract}

PACS: 12.39.Jh, 12.40.Vv, 13.40.Gp, 13.40.Hq

Keywords: Non-relativistic quark models, Roper, electromagnetic decays.

cano@xaloc.ific.uv.es

gonzalep@evalvx.ific.uv.es 
Baryon resonances and in particular the Roper resonance N(1440) deserve special attention at the current moment from the theoretical as well as the experimental point of view. The Roper resonance has been observed at Saturne as an excitation of a hydrogen target scattered by $\alpha$-particles [1]. The transition form factors for the excitation of baryon resonances are going to be systematically measured at TJNAF in a near future. Related experimental programs are carried out in MAMI, ELSA and GRAAL. Theoretically, to predict the precise position of the Roper in the nucleon resonance spectrum and the correct values (including signs) of its strong and electromagnetic transition amplitudes has been a challenge for many years.

Though the simplest image of the Roper corresponds to a radial excitation of the $3 q$ nucleon state, due to the difficulty in describing its phenomenology, 'more completed' or alternative descriptions have been proposed. Among the first ones we can mention the relativized versions of the constituent quark model [2, 3] and the light front constituent quark model [4, 5]. Among the second ones the breathing bag model [6, 7] and the hybrid state descriptions [8, 9, 10, 11] which confer to the Roper an exotic nature. Nonetheless a unified description of the Roper phenomenology has not been achieved and its nature is still under discussion.

In the last few years very precise fits of the low-lying baryon spectrum have been obtained making use of potential models [12, 13, 14]. One of the models 12] has also been succesfully applied to predict the pionic decay widths of the low-lying nucleon and delta resonances [15]. Concerning the Roper, the consideration, via a ${ }^{3} P_{0}$ model, of the $q \bar{q}$ pion structure, becomes essential to correctly reproduce the strong pionic decay data. The purpose of this paper is to apply the same model to the calculation of photo and electroproduction amplitudes of the Roper, the aim being to check whether it is possible or not, from a non-relativistic constituent quark model, to get a unified description of the Roper phenomenology and to learn about its elusive nature.

\section{Photo and electroproduction amplitudes}

Some years ago, Gavela et al. [16] got a reasonable description at that time of the Roper photoproduction amplitude $N(1440) \rightarrow N \gamma$ by combining vector meson dominance (VMD) with a ${ }^{3} P_{0}$ model for the meson production. A simple and immediate generalization to $Q^{2} \neq 0$ is obtained by introducing an intermediate propagator for the meson (a more fundamental, but at the same time more complicated technical approach would be to couple the photon to the $q \bar{q}$ structure of the meson). The transition matrix element for a general process $B \rightarrow B^{\prime} \gamma$ reads: 


$$
\left\langle B^{\prime} \gamma|H| B\right\rangle=\sum_{V}\left\langle B^{\prime} V\left|H_{{ }^{3} P_{0}}\right| B\right\rangle \frac{1}{m_{V}^{2}+Q^{2}}\left\langle\gamma\left|H_{V \gamma}\right| V\right\rangle
$$

with $V=\rho, \omega$ and [17]:

$$
\left\langle\gamma\left|H_{V \gamma}\right| V\right\rangle=e \frac{m_{V}^{2}}{f_{V}}
$$

The matrix element in the ${ }^{3} P_{0}$ model is given by [18]:

$$
\begin{aligned}
\left\langle B^{\prime} V\left|H_{{ }^{3} P_{0}}\right| B\right\rangle= & -3 \gamma \sum_{m}(110 \mid m-m) \\
& \int d \vec{p}_{1} d \vec{p}_{2} d \vec{p}_{3} d \vec{p}_{4} d \vec{p}_{5} \mathcal{Y}_{1 m}\left(\vec{p}_{4}-\vec{p}_{5}\right) \delta\left(\vec{p}_{4}+\vec{p}_{5}\right) \Phi_{\text {Pair }}^{-m} \\
& {\left[\Psi_{B^{\prime}}\left(\vec{p}_{1}, \vec{p}_{2}, \vec{p}_{4}\right) \Phi_{B^{\prime}}\right]^{*}\left[\Psi_{V}\left(\vec{p}_{3}, \vec{p}_{5}\right) \Phi_{V}\right]^{*}\left[\Psi_{B}\left(\vec{p}_{1}, \vec{p}_{2}, \vec{p}_{3}\right) \Phi_{B}\right] }
\end{aligned}
$$

where $\gamma$ is the pair creation coupling constant, $\mathcal{Y}_{1 m}$ a solid harmonic, $\Psi$ stands for the momentum space wave function and $\Phi$ for the spin-isospin wave function.

By using eqs. (2) and (3) we can write after some algebra the transition matrix element as:

$$
\begin{aligned}
\left\langle B^{\prime} \gamma|H| B\right\rangle= & -3 e \gamma\left(\frac{m_{A}}{\omega_{\gamma}}\right)^{1 / 2} \delta^{(3)}\left(\vec{P}_{B}-\vec{P}_{B}{ }^{\prime}-\vec{q}\right)\left(-\frac{1}{\sqrt{4 \pi}} \frac{1}{2} \sqrt{\frac{2}{3}}\right) \\
& {\left[\frac{1}{f_{\omega}} \frac{m_{\omega}^{2}}{m_{\omega}^{2}+Q^{2}}\left\langle\eta_{B^{\prime}} \mid \eta_{B}\right\rangle+\frac{1}{f_{\rho}} \frac{m_{\rho}^{2}}{m_{\rho}^{2}+Q^{2}}\left\langle\eta_{B^{\prime}}\left|\tau_{0}^{(3)}\right| \eta_{B}\right\rangle\right] } \\
& \left\langle\chi_{B^{\prime}}\right| \int d \vec{p}_{\xi_{1}} d \vec{p}_{\xi_{2}} \Psi_{B^{\prime}}^{*}\left(\vec{p}_{\xi_{1}}, \vec{p}_{\xi_{2}}+\sqrt{\frac{2}{3}} \vec{q}\right) \Psi_{V}^{*}\left(-\sqrt{\frac{2}{3}} \vec{p}_{\xi_{2}}+\frac{\vec{P}_{B}}{3}-\frac{\vec{q}}{2}\right) \\
& {\left[\left(\vec{p}_{3}-\vec{q}\right) \vec{\epsilon}^{*}+i \vec{\epsilon}^{*}\left(\vec{\sigma}^{(3)} \times\left(\vec{p}_{3}-\vec{q}\right)\right)\right] \Psi_{B}\left(\vec{p}_{\xi_{1}}, \vec{p}_{\xi_{2}}\right)\left|\chi_{B}\right\rangle }
\end{aligned}
$$

where $m_{A}$ is an average $\rho$ and $\omega$ mass, $\omega_{\gamma}(\vec{q})$ is the photon energy (trimomentum), $\eta$ stands for the isospin wave function, $\chi$ for the spin one, $\left(\vec{\xi}_{1}, \vec{\xi}_{2}\right)$ are the Jacobi coordinates and $\left(\vec{p}_{\xi_{1}}, \vec{p}_{\xi_{2}}\right)$ its associated momenta operators. The polarization of the vector meson has been denoted by $\vec{\epsilon} . \vec{\sigma}$ 's ( $\vec{\tau}$ 's $)$ as usual denote spin (isospin) matrices. The superindex in the spin and isospin operators indicates the quark on which they act.

All the dependence on a specific quark model for the baryon is contained in the baryon wave functions $\Psi_{B}, \Psi_{B^{\prime}}$. For $\Psi_{V}$ we shall take a gaussian form 18]: 


$$
\Psi_{V}(\vec{q})=\frac{2 R_{A}^{3 / 2}}{\pi^{1 / 4}} \exp \left(-\frac{R_{A}^{2}}{2} \vec{q}^{2}\right) Y_{00}(\hat{q})
$$

The value chosen for $R_{A}$ is $2.82 \mathrm{GeV}^{-1}$ [16], quite close to the one obtained from the study of the leptonic decay of $\rho^{0}$ in the quark model. If we assume the $\mathrm{SU}(3)$ constraint, $f_{\omega}=3 f_{\rho}$, the only free parameter is the ratio $\frac{\gamma}{f_{\rho}}$.

\subsection{Nucleon form factors.}

As a first application of expression (四) it is interesting to analyze the form factors of the nucleon for which we use the Breit system of reference. To get the magnetic form factor we take a transverse polarization vector $\vec{\epsilon}_{ \pm}=$ $\mp \frac{1}{\sqrt{2}}(1, \pm i, 0)$ and compare expression (雨) with the corresponding matrix element in terms of the form factors of the nucleon. Thus we get:

$$
\begin{aligned}
G_{M}^{p, n}\left(Q^{2}\right)= & -3 \gamma\left(m_{A}\right)^{1 / 2}\left(-\frac{1}{\sqrt{4 \pi}} \frac{1}{2} \sqrt{\frac{2}{3}}\right)\left(2 M_{N}(2 \pi)^{3 / 2}\right) \\
& {\left[\frac{1}{f_{\omega}} \frac{m_{\omega}^{2}}{m_{\omega}^{2}+Q^{2}}\left\langle\eta_{B^{\prime}}\left(\frac{1}{2}, \pm \frac{1}{2}\right) \mid \eta_{B}\left(\frac{1}{2}, \pm \frac{1}{2}\right)\right\rangle\right.} \\
& \left.+\frac{1}{f_{\rho}} \frac{m_{\rho}^{2}}{m_{\rho}^{2}+Q^{2}}\left\langle\eta_{B^{\prime}}\left(\frac{1}{2}, \pm \frac{1}{2}\right)\left|\tau_{0}^{(3)}\right| \eta_{B}\left(\frac{1}{2}, \pm \frac{1}{2}\right)\right\rangle\right] \\
& \left\langle\chi_{B^{\prime}}\left(\frac{1}{2},-\frac{1}{2}\right)\right| \int d \vec{p}_{\xi_{1}} d \vec{p}_{\xi_{2}} \Psi_{B^{\prime}}^{*}\left(\vec{p}_{\xi_{1}}, \vec{p}_{\xi_{2}}+\sqrt{\frac{2}{3}} \vec{q}\right) \Psi_{V}^{*}\left(-\sqrt{\frac{2}{3}} \vec{p}_{\xi_{2}}-\frac{\vec{q}}{3}\right) \\
& \frac{1}{q}\left[-q \sigma_{-}^{(3)}+p_{3_{z}} \sigma_{-}^{(3)}-p_{3-} \sigma_{z}^{(3)}-p_{3-}\right] \Psi_{B}\left(\vec{p}_{\xi_{1}}, \vec{p}_{\xi_{2}}\right)\left|\chi_{B}\left(\frac{1}{2}, \frac{1}{2}\right)\right\rangle
\end{aligned}
$$

$M_{N}$ being the nucleon mass.

The electric form factor is given in terms of the time component of the current $\left(G_{E}\left(Q^{2}\right)=\left\langle j_{0}\right\rangle\right)$ that can be related to the spatial one $j_{3}$ by gauge invariance $\left(j_{0}=\frac{q}{\omega} j_{3}, \vec{q} \equiv(0,0, q)\right)$. Then:

$$
\begin{aligned}
G_{E}^{p, n}\left(Q^{2}\right)= & -3 \gamma\left(m_{A}\right)^{1 / 2}\left(-\frac{1}{\sqrt{4 \pi}} \frac{1}{2} \sqrt{\frac{2}{3}}\right)\left(-\sqrt{2}(2 \pi)^{3 / 2}\right) \\
& {\left[\frac{1}{m_{\omega} f_{\omega}} \frac{m_{\omega}^{2}}{m_{\omega}^{2}+Q^{2}}\left\langle\eta_{B^{\prime}}\left(\frac{1}{2}, \pm \frac{1}{2}\right) \mid \eta_{B}\left(\frac{1}{2}, \pm \frac{1}{2}\right)\right\rangle\right.} \\
& \left.+\frac{1}{m_{\rho} f_{\rho}} \frac{m_{\rho}^{2}}{m_{\rho}^{2}+Q^{2}}\left\langle\eta_{B^{\prime}}\left(\frac{1}{2}, \pm \frac{1}{2}\right)\left|\tau_{0}^{(3)}\right| \eta_{B}\left(\frac{1}{2}, \pm \frac{1}{2}\right)\right\rangle\right]
\end{aligned}
$$




$$
\begin{aligned}
& \left\langle\chi_{B^{\prime}}\right| \int d \vec{p}_{\xi_{1}} d \vec{p}_{\xi_{2}} \Psi_{B^{\prime}}^{*}\left(\vec{p}_{\xi_{1}}, \vec{p}_{\xi_{2}}+\sqrt{\frac{2}{3}} \vec{q}\right) \Psi_{V}^{*}\left(-\sqrt{\frac{2}{3}} \vec{p}_{\xi_{2}}-\frac{\vec{q}}{3}\right) \\
& q\left[p_{3_{z}}-q+\sigma_{+}^{(3)} p_{3-}-\sigma_{-}^{(3)} p_{3+}\right] \Psi_{B}\left(\vec{p}_{\xi_{1}}, \vec{p}_{\xi_{2}}\right)\left|\chi_{B}\right\rangle
\end{aligned}
$$

Symbolically $\left\langle\chi_{B^{\prime}}\left|\sigma_{-}^{(3)}\right| \chi_{B}\right\rangle G_{E}\left(Q^{2}\right) \propto Q^{2} G_{M}\left(Q^{2}\right)$. Let us note that the factor $q$ multiplying the last bracket makes the electric form factor to vanish at $Q^{2}=0$, what can be interpreted as an indication that the total charge of the mesonic cloud $(\rho, \omega)$ in the nucleon is zero. Therefore, in order to get the baryon charge for $G_{E}^{p}\left(Q^{2}=0\right)$, VMD can not be the only mechanism for the photon-baryon interaction.

We propose instead an extended vector meson dominance model (EVMD) as schematically pictured in fig. 1. The first diagram on the r.h.s. corresponds to VMD as calculated above. The second diagram on the r.h.s. corresponds to a direct coupling to the baryon for which we shall use a modified elementary emission model [19] where a convergent $(p / E)$ instead of a $(p / m)$ expansion has been applied [20]. Quite similar assumptions underlie the so called two-phase models 21] where the electromagnetic properties of the nucleon come from two sources, the vector meson cloud and the core of quarks respectively corresponding to the two diagrams we consider in fig. 1. Hence we can summarize the EVMD scenario in the equations:

$$
\begin{aligned}
G_{E}^{p, n}\left(Q^{2}\right) & =\left[G_{E}^{p, n}\left(Q^{2}\right)\right]_{\mathrm{eem}}+\left[G_{E}^{p, n}\left(Q^{2}\right)\right]_{\mathrm{vmd}} \\
G_{M}^{p, n}\left(Q^{2}\right) & =c\left[G_{M}^{p, n}\left(Q^{2}\right)\right]_{\mathrm{eem}}+\left[G_{M}^{p, n}\left(Q^{2}\right)\right]_{\mathrm{vmd}}
\end{aligned}
$$

where the relative weight parameter of the two diagrams $c$ plays the same role as an anomalous magnetic moment of the quarks. Note that although the value of the quark mass used is $m_{q} \approx \frac{M_{N}}{3}$, due to the $(p / E)$ expansion in the EEM, the magnetic moment is not saturated by $\left[G_{M}^{p, n}\left(Q^{2}=0\right)\right]_{\mathrm{eem}}$, the remaining contribution having to be provided by $\left[G_{M}^{p, n}\left(Q^{2}=0\right)\right]_{\mathrm{vmd}}$. For the electric form factor the relative weight is 1 due to the charge conservation (recall that $\left.\left[G_{E}^{p, n}\left(Q^{2}=0\right)\right]_{\mathrm{vmd}}=0\right)$.

To fix the two parameters $\frac{\gamma}{f_{\rho}}$ and $c$, we can use the magnetic moment $\mu_{p}$ and the square mean radius of the proton $\left(\left\langle r_{p}^{2}\right\rangle\right)$ for instance. One should be aware however that important contributions to $\left\langle r_{p}^{2}\right\rangle$, as the one from the Darwin-Foldy term $\approx\left(\frac{2}{3 m_{q}^{2}}\right)$, have not been considered. Actually, a value of $\left\langle r_{p}^{2}\right\rangle$ between 0.5 and $0.6 \mathrm{fm}^{2}$ seems to be a better choice to get a very good fit of most electromagnetic transitions [22].

In order to extract some results we shall make use of two potential models $V_{\mathrm{I}}, V_{\mathrm{II}}$, extensively detailed elsewhere 15. For the sake of completeness we only quote here their expressions (values of the parameters as in ref. [15]): 


$$
\begin{aligned}
V_{\mathrm{I}} & =\sum_{i<j} \frac{1}{2}\left[\frac{r_{i j}}{a^{2}}-\frac{\kappa}{r_{i j}}+\frac{\kappa}{m_{i} m_{j}} \frac{\exp \left(-r_{i j} / r_{0}\right)}{r_{0}^{2} r_{i j}} \vec{\sigma}_{i} \vec{\sigma}_{j}-D\right] \\
V_{\mathrm{II}} & =\sum_{i<j} \frac{1}{2}\left[\frac{r_{i j}}{a^{2}}-\frac{\kappa}{r_{i j}}+\frac{\kappa}{6 m_{i} m_{j}} \frac{\exp \left(-r_{i j} / r_{0}\right)}{r_{0}^{2} r_{i j}} \vec{\sigma}_{i} \vec{\sigma}_{j}-D\right] \\
& +\sum_{i \neq j \neq k \neq i} \frac{1}{2} \frac{V_{0}}{m_{i} m_{j} m_{k}} \frac{e^{-m_{0} r_{i j}}}{m_{0} r_{i j}} \frac{e^{-m_{0} r_{i k}}}{m_{0} r_{i k}}
\end{aligned}
$$

$V_{\text {I }}$ contains the 'minimal' one gluon exchange kind of potential and provides a reasonable average fit for the meson and baryon spectra (not fitting the Roper in the spectrum) [23] whereas $V_{\mathrm{II}}$, that incorporates a phenomenological threequark interaction, fits very precisely the low lying baryon spectrum [12] and most of its pionic strong decay widths, in particular the Roper resonance $N(1440)$ mass and decay widths [20].

By fixing $\mu_{p}=2.79$ and $\left\langle r_{p}^{2}\right\rangle=0.54 \mathrm{fm}^{2}$ one gets $c=0.49(0.17)$ and $\frac{\gamma}{f_{\rho}}=-0.38(-0.71)$ for $V_{\mathrm{I}}\left(V_{\mathrm{II}}\right)$. Then for the neutron we get $\mu_{n}=-1.82$ $(-1.84)$ and $\left\langle r_{n}^{2}\right\rangle=-0.003(-0.002)$ for $V_{\mathrm{I}}\left(V_{\mathrm{II}}\right)$. The separate contributions from the two diagrams are shown in table 1.

Let us emphasize that we do not do a completely consistent construction of the transition operator according to the mass operator. We assume that the wave functions we obtain by solving the Schrödinger equation for $V_{\mathrm{I}}$ of $V_{\mathrm{II}}$ represent, through the effective parameters and interactions of the potential a sort of averaged $(p / E)$ quark core wave function. One should realize however that the corrections introduced by the $(p / E)$ expansion in the transition operator get correlated to the model wave functions through the weight parameter.

It is ilustrative to draw the magnetic form factor. Looking at fig. 2 it is clear that the data lie between the predictions of the two models. This emphasizes the major role of the form of the transition operator (as it was the case in the strong decays of the Roper where the $q \bar{q}$ structure of the pion was essential) leaving a fine tunning effect to the specific quark potential model employed. In particular, the ${ }^{3} P_{0}$ structure is mostly responsible to get the tendency of experimental data.

\subsection{Roper transition amplitudes.}

The Roper transition amplitudes calculated in the EVMD model are shown in figs. 3.a and 3.b. Though a clarification of experimental data seems to be necessary, it is worth to emphasize that we reproduce the experimental sign of the transverse amplitude at $Q^{2}=0$ (contrary to what happens with an EEM calculation) for what the VMD as provided by the ${ }^{3} P_{0}$ model becomes essential 
(see table 1). The smallness of the EEM contribution is related to the use of a $(p / E)$ expansion instead of a $(p / m)$ one, combined with the presence of a node in the Roper wave function (note the higher sensitivity of this process as compared to the magnetic nucleon form factor case though the expression for the amplitude is similar). Furthermore, we predict a change of sign at $Q^{2} \approx 0.5-1 \mathrm{GeV}^{2}$, a feature suggested by the present data. The behaviour of our amplitude resembles the one obtained with a light-front formalism [4, 5], though we do not have a significant configuration mixing as it was the case in ref. [4.

In order to have a deeper understanding of the nature of the Roper a test based on the calculation of the longitudinal amplitudes was proposed [11]. In figs. 3.c and 3.d we show our results for $S_{1 / 2}$. The interpretation of experimental points [26] in terms of the longitudinal amplitude is controversial (see for instance refs. [4] and [11]) about the sign of this amplitude. Then no definitive conclusion shoud be extracted before more confident data are available and agreement about its interpretation is reached. Meantime we may conclude that a $3 q$ structure of the Roper cannot be discarded once the transition operator incorporates the physically relevant ingredients.

The resulting picture of the Roper is that of a small quark core dressed by a mesonic cloud of total charge zero. The contribution of the cloud to the baryon masses can be taken into account quite approximately, through the values of the effective parameters or the potential, in a $3 q$ state. For the transitions, the cloud contribution has to be considered explicitely (as an explicit $|q q q q \bar{q}\rangle$ component or in the transition operator as we have done). No exotic explanation of the Roper nature seems to be needed, reinforcing the similar conclusions obtained in different frameworks [4, 5].

The analysis can be extended to other resonances [22]. In particular, for the $\Delta(1600)$, the Roper of the $\Delta$, the $Q^{2}$ behaviour of the amplitude is depicted in fig. 4 , showing a much more pronounced quark model dependence. In our model it also corresponds to a radial excitation so the comparison to data when available may be a more stringent test about our conclusions.

We are grateful to S. Singh and S. Noguera for their suggestions and comments and to G. Salmè for his immediate answer and clarification to our questions on the subject. This work has been partially supported by DGES under grants PB95-1096, PB94-0080 and by EC-TMR network HaPHEEP under contract ERBTMRX CT96-0008.

\section{References}

[1] H.P. Morsch et al., Phys. Rev. Lett. 69 (1992) 1336. 
[2] S. Capstick, Phys. Rev. D46 (1992) 1965; Phys. Rev. D46 (1992) 2864.

[3] F.E. Close and Z. Li, Phys. Rev. D42 (1990) 2194. Z. Li and F.E. Close Phys. Rev. D42 (1990) 2207.

[4] F. Cardarelli, E. Pace, G. Salmè and S. Simula, Phys. Lett. B397 (1997) 13.

[5] S. Capstick and B.D. Keister, Phys. Rev. D51 (1995) 3598.

[6] G.E. Brown, J.W. Durso and M.B. Johnson, Nucl. Phys. A39 (1983) 447.

[7] P.A.M. Guichon, Phys. Lett. B164 (1985) 361.

[8] T. Barnes and F.E. Close, Phys. Lett. B123 (1983) 89.

[9] F.E. Close, Rep. Prog. Phys. 51 (1988) 833. F.E. Close, Nucl. Phys. A497 (1989) 109c.

[10] C.E. Carlson and N.C. Mukhopadhyay, Phys. Rev. Lett. 67 (1991) 3745.

[11] Z. Li, V. Burkert and Z. Li, Phys. Rev. D46 (1992) 70.

[12] B. Desplanques, C. Gignoux, B. Silvestre-Brac, P. González, J. Navarro and S. Noguera, Z. Phys. A343 (1992) 331. A. Valcarce, F. Fernández, P. González and V. Vento, Phys. Lett. B367 (1996) 35.

[13] Y.B. Dong, J.C. Su and S.S. Wu, J. Phys. G20 (1994) 73.

[14] L.Ya. Glozman and D.O. Riska, Phys. Rep. 268 (1996) 263.

[15] F. Cano, P. González, S. Noguera and B. Desplanques, Nucl. Phys. A603 (1996) 257.

[16] M.B. Gavela, A. Le Yaouanc, L. Oliver, O. Pène, J.-C. Raynal and S. Sood, Phys. Rev. D21 (1980) 182.

[17] J.J. Sakurai, Currents and Mesons (The University of Chicago Press, 1969).

[18] A. Le Yaouanc, L. Oliver, O. Pène and J.-C. Raynal, Phys. Rev. D8 (1973) 2223; Phys. Rev. D11 (1975) 1272.

[19] A. Le Yaouanc, Ll. Oliver, O. Pène and J.-C. Raynal, Hadron Transitions in the Quark Model (Gordon and Breach Science Publishers, 1988).

[20] F. Cano, P. González, B. Desplanques and S. Noguera, Z. Phys. A359 (1997) 315. 
[21] G.E. Brown, M. Rho and W. Weise, Nucl. Phys. A454 (1986) 669.

[22] F. Cano, P. González, B. Desplanques and S. Noguera, work in preparation.

[23] B. Silvestre-Brac and C. Gignoux, Phys. Rev. D32 (1985) 743.

[24] W. Bartel et al., Nucl. Phys. B58 (1973) 429. R.G. Arnold et al., Phys. Rev. Lett. 57 (1986) 174. P.E. Bosted et al., Phys. Rev. Lett. 68 (1992) 3841.

[25] Particle Data Group, Phys. Rev. D54 (1996) 1.

[26] Ch. Gerhardt, Z. Phys C4 (1980) 311. 


\section{Table caption}

Table 1 Charge radii (in $\mathrm{fm}^{2}$ ) and magnetic moments (in nuclear magnetons) for the nucleon in the two models $V_{\mathrm{I}}$ and $V_{\mathrm{II}}$. The contribution from each diagram in fig. 2 is shown. The photoproduction amplitudes $A_{1 / 2}^{p, n}, S_{1 / 2}^{p, n}$ (in units of $10^{-3} \mathrm{GeV}^{-1 / 2}$ ) correspond to the process $N(1440) \rightarrow N \gamma$. The EEM contribution includes the relative weight $c$.

\section{Figure captions}

Figure 1 Extended vector meson dominance picture.

Figure 2. Magnetic form factor for the proton calculated in the EVMD model for the two potentials $V_{\mathrm{I}}$ (solid line), $V_{\mathrm{II}}$ (dashed line). For exp. data see for instance [24]

Figure 3. Transverse (a,b) and longitudinal (c,d) electroproduction amplitudes for $\mathrm{N}(1440)$. The solid line corresponds to $V_{\mathrm{I}}$ and the dashed line to $V_{\mathrm{II}}$. Experimental points at $Q^{2}=0$ in a) and b) are taken from [25]. For other experimental points see [11].

Figure 4. Transverse electroproduction amplitude for the process $\Delta^{+}(1600) \rightarrow p \gamma$. Notation as in fig. 3 . 


\begin{tabular}{|c|rrr|rrr|c|}
\hline \hline & \multicolumn{3}{|c|}{$V_{\mathrm{I}}$} & \multicolumn{3}{|c|}{$V_{\mathrm{II}}$} & \multirow{2}{*}{ Exp. } \\
\cline { 2 - 6 } & EEM & VMD & Total & EEM & VMD & Total & \\
\hline $\begin{array}{c}\left\langle r_{p}^{2}\right\rangle \\
\left\langle r_{n}^{2}\right\rangle\end{array}$ & 0.238 & 0.302 & 0.54 & 0.133 & 0.407 & 0.54 & $0.74 \pm 0.02$ \\
\hline$\mu_{p}$ & 0.93 & -0.012 & -0.032 & -0.005 & -0.01 & -0.015 & $-0.119 \pm 0.004$ \\
$\mu_{n}$ & -0.61 & -1.21 & -1.82 & -0.18 & -1.66 & -1.84 & -1.91 \\
\hline$A_{1 / 2}^{p}$ & 0.63 & -82.1 & -81.5 & -1.9 & -164 & -166 & $-65 \pm 4$ \\
$A_{1 / 2}^{n}$ & -.26 & 52.3 & 52.0 & 1.3 & 108 & 110 & $40 \pm 10$ \\
\hline$-S_{1 / 2}^{p}$ & -16.5 & -30.3 & -46.8 & -4.7 & -61.4 & -66.1 & - \\
$-S_{1 / 2}^{n}$ & 2.1 & 3.7 & 5.9 & 0.25 & 2.6 & 2.8 & - \\
\hline \hline
\end{tabular}

Table 1 


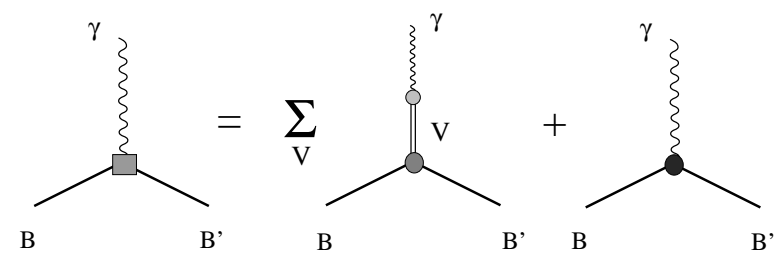

fig. 1

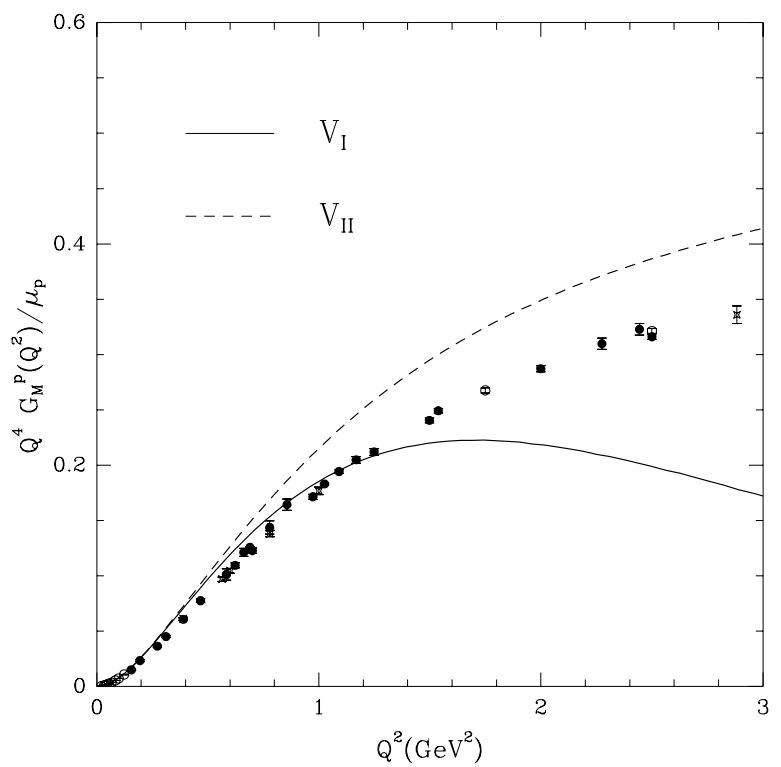

fig. 2 


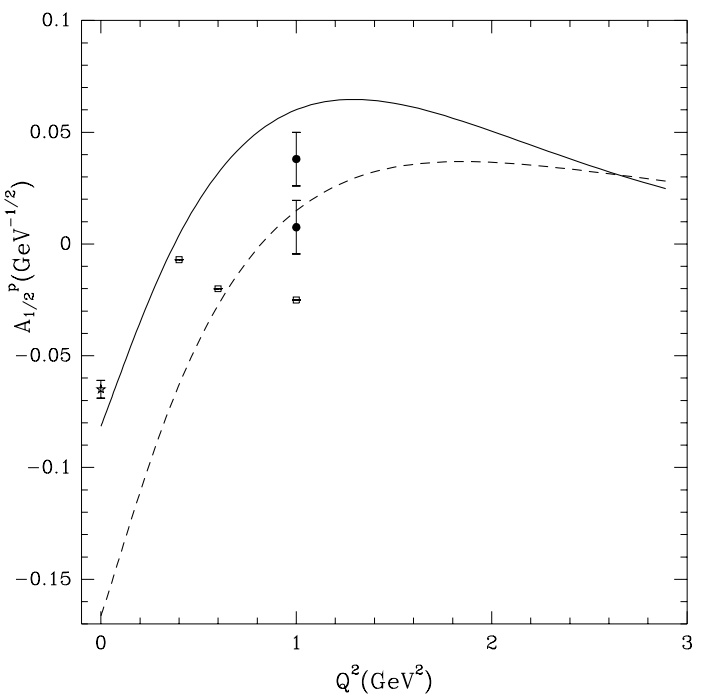

fig. 3a

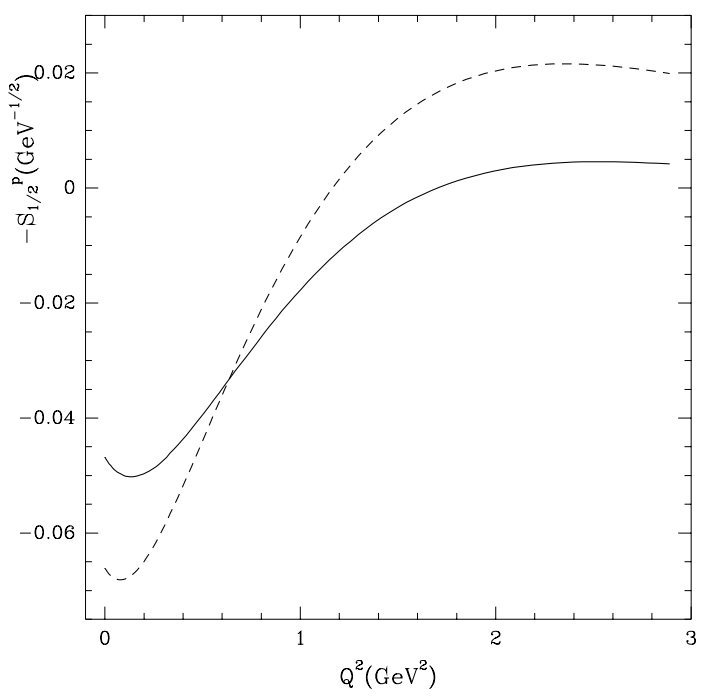

fig. 3c

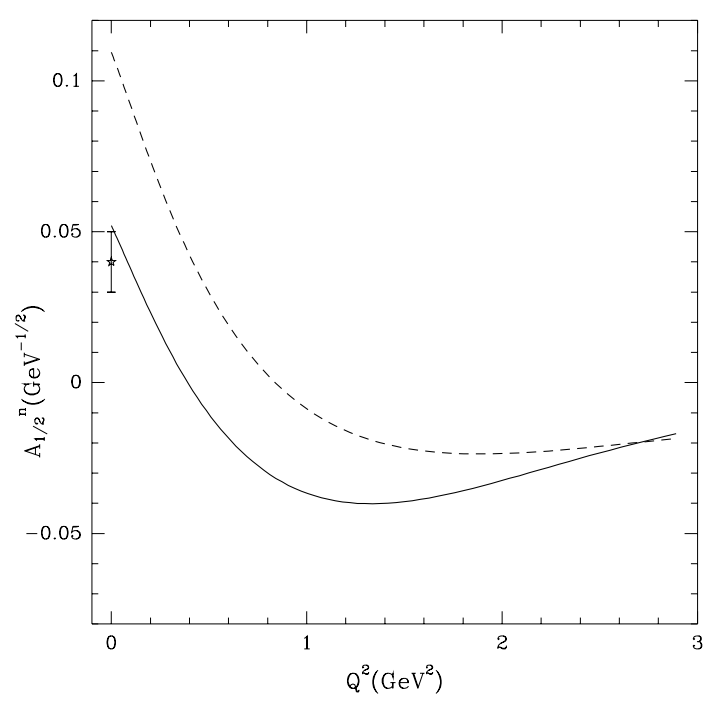

fig. $3 \mathrm{~b}$

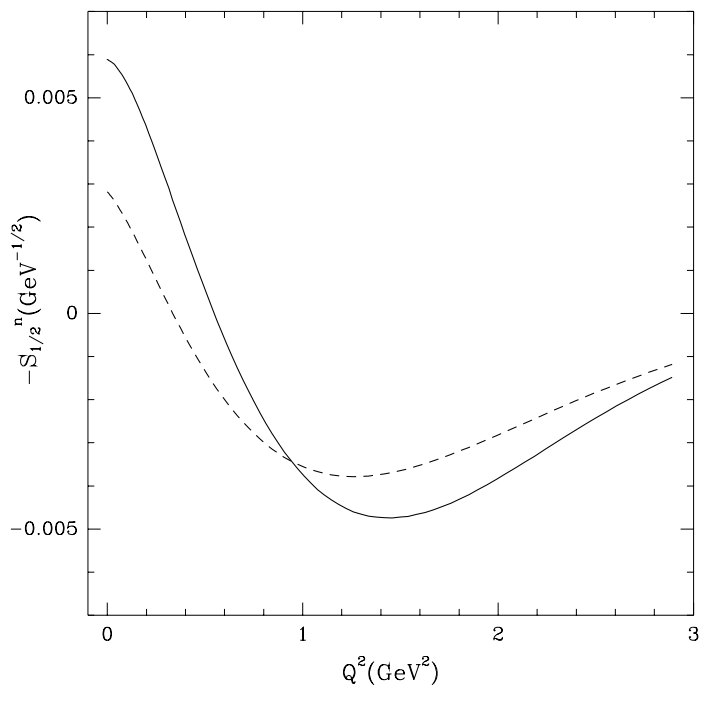

fig. 3d 


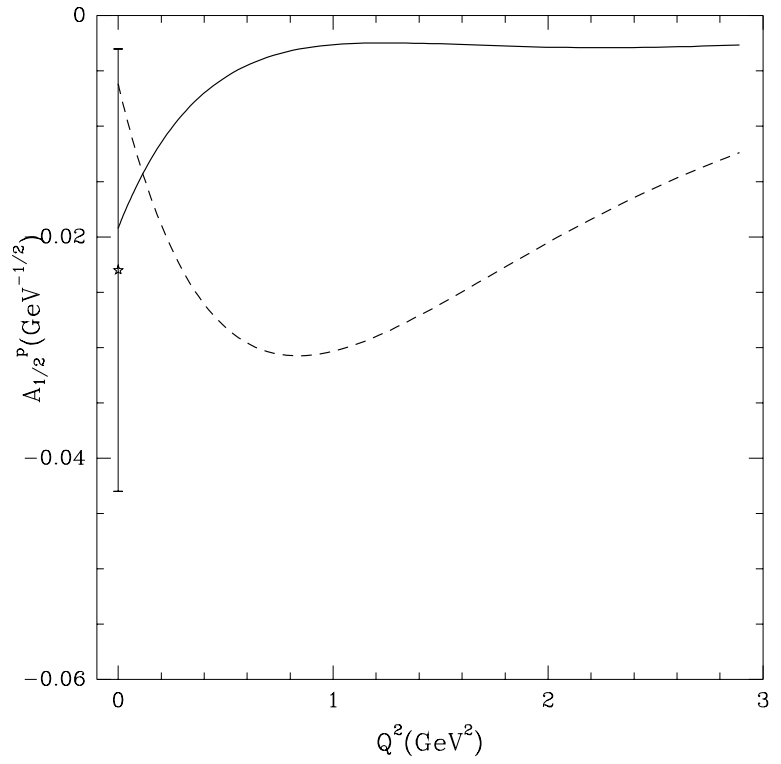

fig. 4 